\title{
Reduced Aging in Carbon Molecular Sieve Membranes Derived From PIM-1 and MOP-18
}

Whitney K. Cosey, Kenneth J. Balkus, Jr., John P. Ferraris, Inga H. Musselman*

Department of Chemistry and Biochemistry, The University of Texas at Dallas, 800 W. Campbell Road, Richardson, TX 75080, USA

\section{Table of Contents}

Supplementary Characterization ..........................................................................

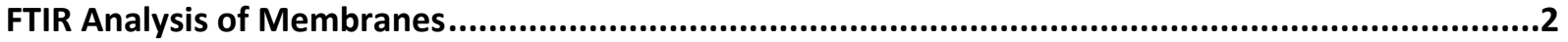

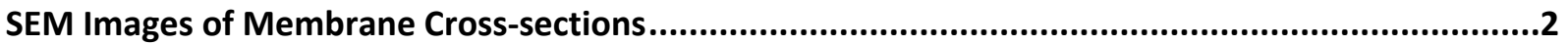

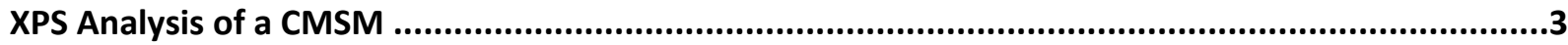

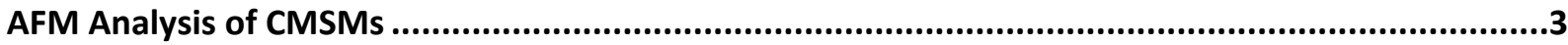

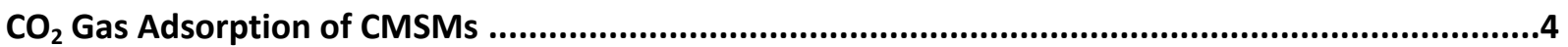




\section{Supplementary Characterization}

\section{FTIR Analysis of Membranes}

ATR-FTIR spectroscopy (Figure S1) was used to confirm the incorporation of MOP-18 into the polymeric precursor PIM-1. The $40 \mathrm{wt} / \mathrm{wt}$ MOP-18/PIM-1 MMM spectra were normalized to the C-O alkoxy stretch at $1000 \mathrm{~cm}^{-1}$ associated with PIM-1. Pristine PIM-1 shows an intense peak at $2240 \mathrm{~cm}^{-1}$ corresponding to the $-\mathrm{CN}$ functional group present in the backbone. This peak is also observed in the mixed-matrix membrane containing $40 \mathrm{wt} / \mathrm{wt} \mathrm{MOP-18}$ (Figure S1b). The retention of this peak indicates that there is no modification to the polymer precursor in the form of crosslinking due to the incorporation of the additive. The two peaks present at 1580 and 1630 $\mathrm{cm}^{-1}$ were used to track the $\mathrm{C}=\mathrm{C}$ stretch associated with the MOP-18 linker (Figure S1c). These two peaks as well as the peak present at $730 \mathrm{~cm}^{-1}$, which is associated with the $\mathrm{C}-\mathrm{O}$ present in copper acetate (Figure S1d), indicate a successful incorporation of the additive.
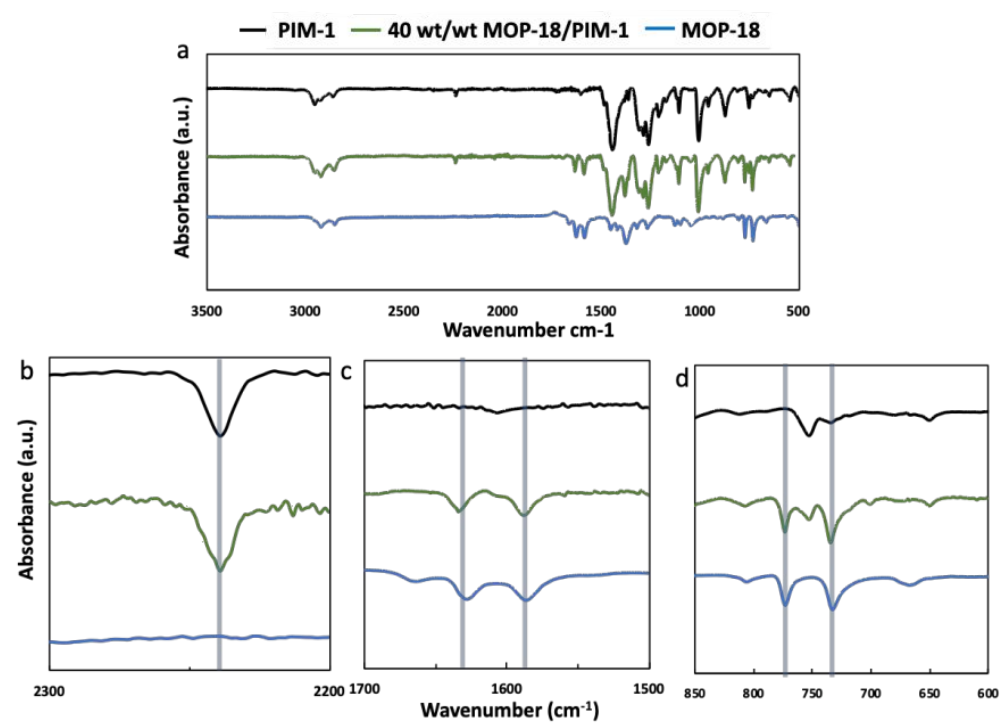

Figure S1: ATR-FTIR spectra of membranes: (a) Full spectra of PIM-1 (black), 40 wt/wt MOP18/PIM-1 MMM (green), and MOP-18 powder (blue) along with insets, (b) -CN stretch, (c) C=C alkene stretch, and (d) C-O stretch.

\section{SEM Images of Membrane Cross-sections}

Images obtained of PIM-1 and the 40 wt/wt MOP-18/PIM-1 MMM using SEM are shown in Figure S2. The nodular morphology of the pristine PIM-1 polymer cross-section remains intact upon the incorporation of the MOP-18. This is due to the strong interaction between the additive and the polymer. 


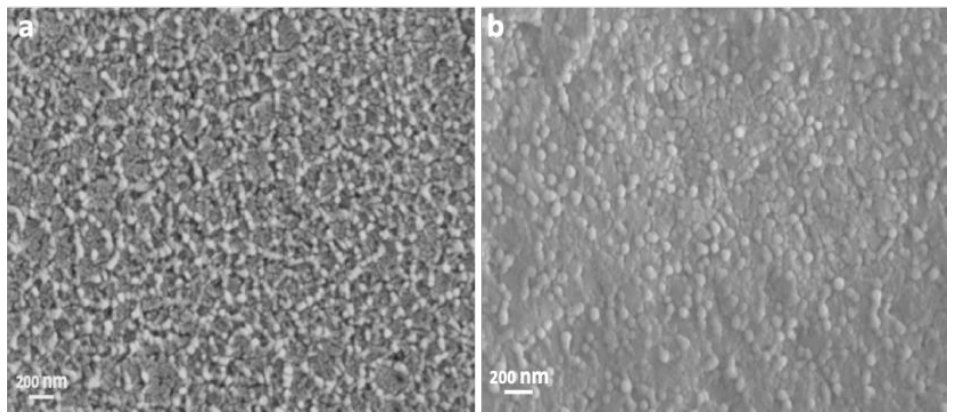

Figure S2. SEM images of polymer membrane cross-sections: (a) PIM-1, and (b) 40 wt/wt MOP18/PIM-1 MMM.

\section{XPS Analysis of a CMSM}

XPS analysis of the 40 wt/wt MOP-18/PIM-1 CMSM (Figure S3) showed two peaks at 932.7 and $952.5 \mathrm{eV}$, corresponding to copper metal. The lack of the satellite peak in the range of 940-950 $\mathrm{eV}$, commonly associated with $\mathrm{CuO}$, confirms the formation of copper metal and not copper oxide.
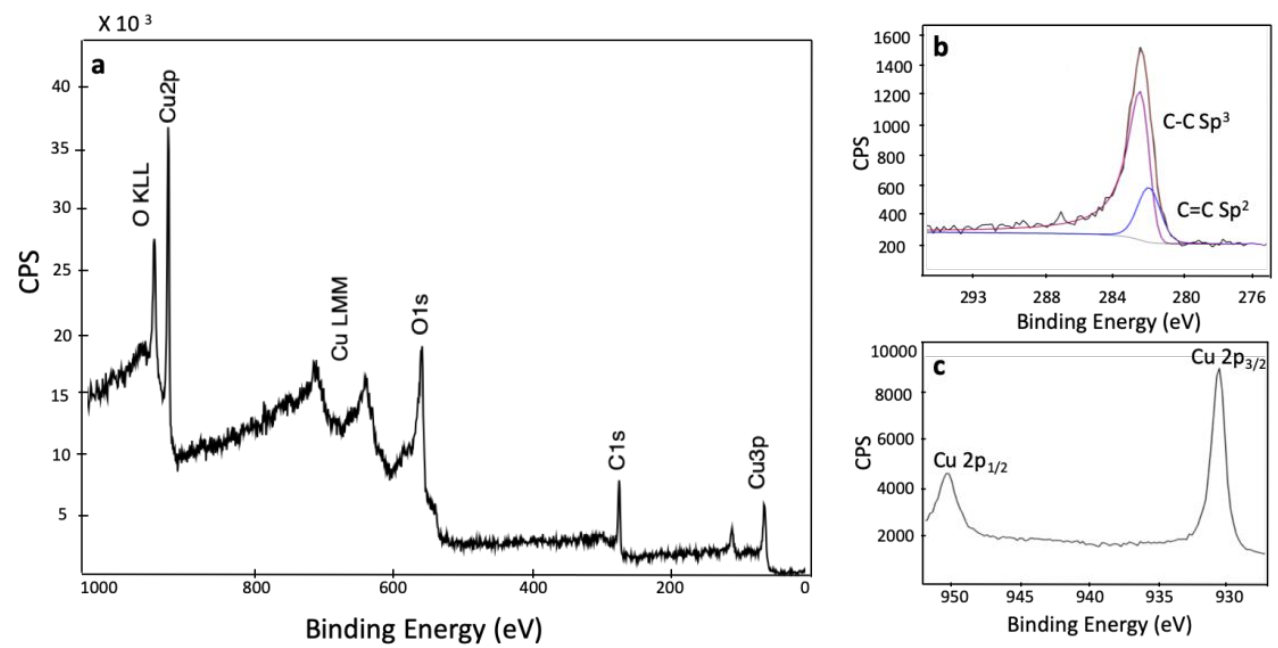

Figure S3. XPS spectra of 40 wt/wt MOP-18/PIM-1 CMSM: (a) survey, (b) carbon, and (c) copper.

\section{AFM Analysis of CMSMs}

The mechanical properties of CMSMs is a topic of concern for membranologists, however there are few studies which investigate them due to the inherent brittleness of CMSMs. AFM was used to analyze the carbon membranes in this study, and it was observed that the incorporation of MOP-18 into PIM-1 does in fact increase the CMSMs Young's modulus. Figure S4 shows images acquired for a PIM-1 CMSM (Figure S4a) and a $40 \mathrm{wt} / \mathrm{wt}$ MOP-18/PIM-1 derived CMSM (Figure S4b). The Young's modulus increases 2-fold, from 3.47 to $6.33 \mathrm{GPa}$, for 
the respective CMSMs. This increase is attributed to the copper as well as to the residual carbon from MOP-18 following pyrolysis, which improved the CMSM mechanical properties.
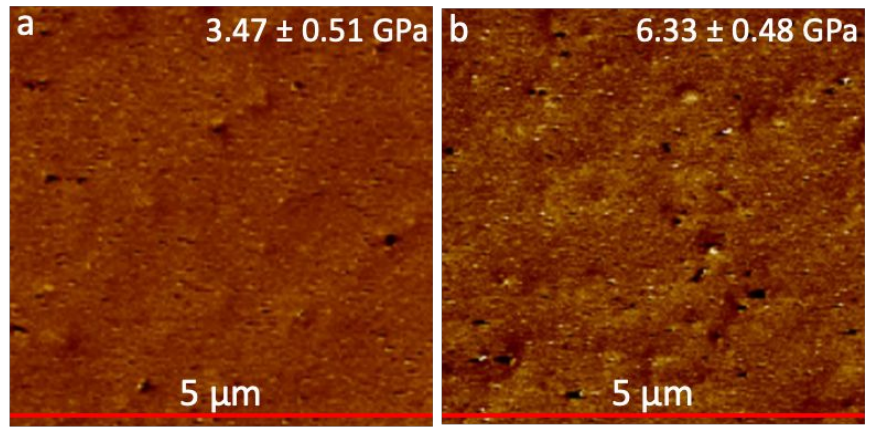

Figure S4: AFM images of (a) PIM-1 CMSM and (b) 40 wt/wt MOP-18/PIM-1 derived CMSM.

\section{$\mathrm{CO}_{2}$ Gas Adsorption of CMSMs}

Measuring the pore size distribution of CMSMs before and after aging can illustrate the changes that occur due to physical aging. The incorporations of Cu pillars, which aim to prevent the collapse of the membranes micropore structure, may also be investigated by measuring the pore size of a Cu-pillared CMSM before and after aging. Isotherms are presented in Figure S5 for fresh and 21-d aged CMSMs, along with their corresponding pore size distributions. It is observed that the 21-d aged PIM-1 CMSM increases in $\mathrm{CO}_{2}$ adsorption, whereas the aged $\mathrm{Cu}$ pillared CMSM remains constant. The increase in $\mathrm{CO}_{2}$ adsorption corresponds to an increase in the CMSMs ultramicropores. The increase is due to the collapse of the CMSMs micropores, which generates ultramicropores. The results demonstrate that the Cu-pillared PIM-1 CMSM retained its "fresh" pore size distribution due to the incorporation of Cu pillars.

—Aged 40 wt/wt MOP-18/PIM-1 — Fresh 40 wt/wt MOP-18/PIM-1 — Aged PIM-1 — Fresh PIM-1
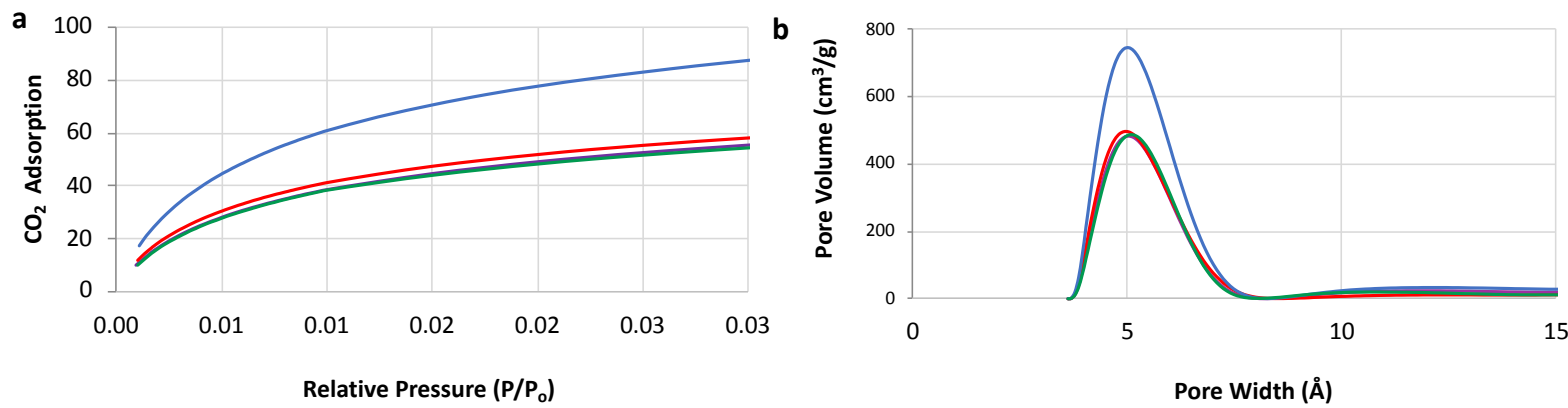

Figure S5: (a) Plot of $\mathrm{CO}_{2}$ adsorption at $0{ }^{\circ} \mathrm{C}$ versus relative pressure for PIM-1-derived CMSMs, and (b) their corresponding pore size distributions calculated from the 2D-NLDFT. 